\title{
The Local Environments of Core-Collapse SNe within Host Galaxies
}

\section{Joseph P Anderson ${ }^{1}$, Stacey M Habergham ${ }^{2}$, Phil A James ${ }^{2} \&$ M Hamuy ${ }^{1}$}

\author{
${ }^{1}$ Departamento de Astronomía, Universidad de Chile, Casilla 36-D, Santiago, Chile \\ ${ }^{2}$ Astrophysics Research Institute, Liverpool John Moores University, Twelve Quays House, \\ Egerton Wharf, Birkenhead, CH41 1LD \\ email: anderson@das.uchile.cl
}

\begin{abstract}
We present constraints on core-collapse supernova progenitors through observations of their environments within host galaxies. This is achieved through 2 routes. Firstly, we investigate the spatial correlation of supernovae with host galaxy star formation using pixel statistics. We find that the main supernova types form a sequence of increasing association to star formation. The most logical interpretation is that this implies an increasing progenitor mass sequence going from the supernova type Ia arising from the lowest mass, through the type II, type Ib, and the supernova type Ic arising from the highest mass progenitors. We find the surprising result that the supernova type IIn show a lower association to star formation than type IIPs, implying lower mass progenitors. Secondly, we use host HII region spectroscopy to investigate differences in environment metallicity between different core-collapse types. We find that supernovae of types Ibc arise in slightly higher metallicity environments than type II events. However, this difference is not significant, implying that progenitor metallicity does not play a dominant role in deciding supernova type.
\end{abstract}

Keywords. (stars:) supernovae: general, (ISM:) HII regions

\section{Introduction}

Mapping the links between progenitors and observed transients has become a key goal of supernova (SN) science, helping us to understand stellar evolution processes while also strengthening our confidence in the use of SNe to understand the Universe. In a number of cases the progenitor stars of core-collapse (CC) SNe have been directly identified on pre-explosion imaging (e.g. Smartt 2009). This allows one to gain 'direct' knowledge of progenitor masses when observed luminosities are compared to stellar models. However, the rarity of such detections limits the statistical results which can be gained. One can also relate overall host galaxy properties to the relative rates of SNe to infer progenitor properties (e.g. Prieto, Stanek \& Beacom 2008, Boissier \& Prantzos 2009). While this can attain significant statistics, the presence of multiple stellar populations within galaxies complicates the implications which can be drawn. Our method is intermediate to these; we investigate differences in CC SN progenitor characteristics using observations of the exact environments at the discovery positions of their explosions. Other recent examples using similar techniques include: Leloudas et al. (2010), Modjaz et al. (2011) and Kelly \& Kirshner (2011). 

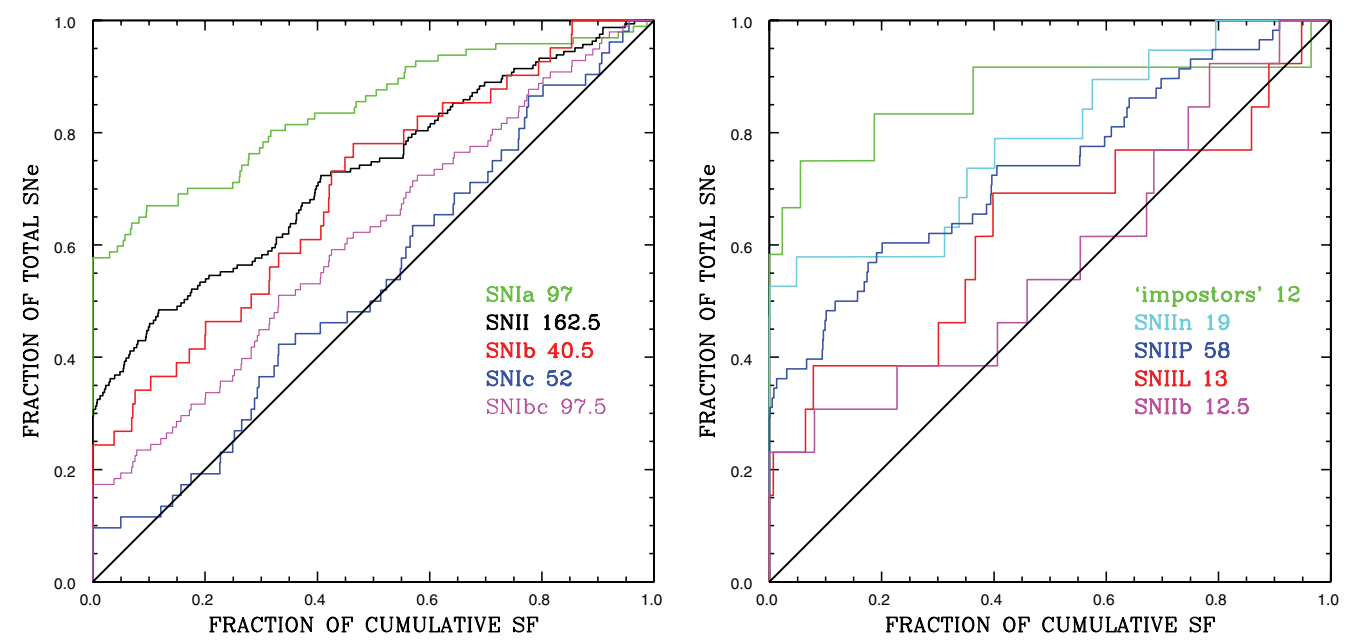

Figure 1. Left a): cumulative pixel statistics of the main SN types. SNIa are in green, SNII black, SNIb red and SNIc blue (combined SNIbc magenta). The black diagonal line illustrates a flat distribution that accurately traces the SF. As a distribution moves away to the left from this diagonal it displays a lower association with SF. Right b): Same as a) but for SNII sub-types. 'Impostors' are shown in green, SNIIn cyan, SNIIP blue, SNIIL red, and SNIIb magenta.

\section{Spatial correlations with star formation}

We have obtained host galaxy $\mathrm{H} \alpha$ imaging for $260 \mathrm{CC}$ SNe. The sample can be separated into 58 SNIIP, 13 IIL, 12.5 IIb (one object has indistinct type), 19 IIn, 12 'impostors' plus 48 SNII (no sub-type), and $40.5 \mathrm{SNIb}, 52 \mathrm{SNIc}$ plus $5 \mathrm{SNIb} / \mathrm{c}$. To analyse the association of each SN with the SF of its host galaxy we use our 'NCR' (Normalised Cumulative Rank pixel function) pixel statistic presented in James \& Anderson (2006). To produce an NCR value for each SN we proceed with the following steps. First the sky-subtracted $\mathrm{H} \alpha$ image pixels are ordered into a rank of increasing pixel count. Alongside this we form the cumulative distribution of this ranked increasing pixel count. Negative cumulative values are then set to zero and all other values are normalised to the total flux summed over all pixels. It follows that each pixel has a value between 0 and 1 , where values of 0 correspond to zero flux values while a value of 1 means the pixel has the highest count within the image. We measure an NCR value for each SN within our sample and proceed to build distributions for each CC SN type. The resulting cumulative distributions are displayed in Figure 1. In both of these figures ( $a$ and b), as a distribution moves closer to the black diagonal line from the top left hand side of the plots, it is showing a higher correlation with bright HII regions. In Fig. 1a) we see a clear sequence of increasing association to SF going from the SNIa, through the SNII, the SNIb and finally the SNIc. The most logical way to interpret differences in associations to SF is that more massive stars will be more highly correlated with bright HII regions. This is because these stars are a) more likely to produce sufficient ionizing flux to produce bright HII regions, and b) they will have shorter lifetimes and therefore have less time to move away from host HII regions. The implication is then that we observe a sequence of increasing progenitor mass. This starts with the SNIa arising from the lowest mass, followed by the SNII, then the SNIb, and finally the SNIc arising from the most massive progenitors. These trends were first seen in a smaller sample published in Anderson \& James (2008). However, the current data set is the first to clearly separate the SNIb from the SNIc, with the latter now being seen to be significantly more associated with SF, and hence arising from higher 

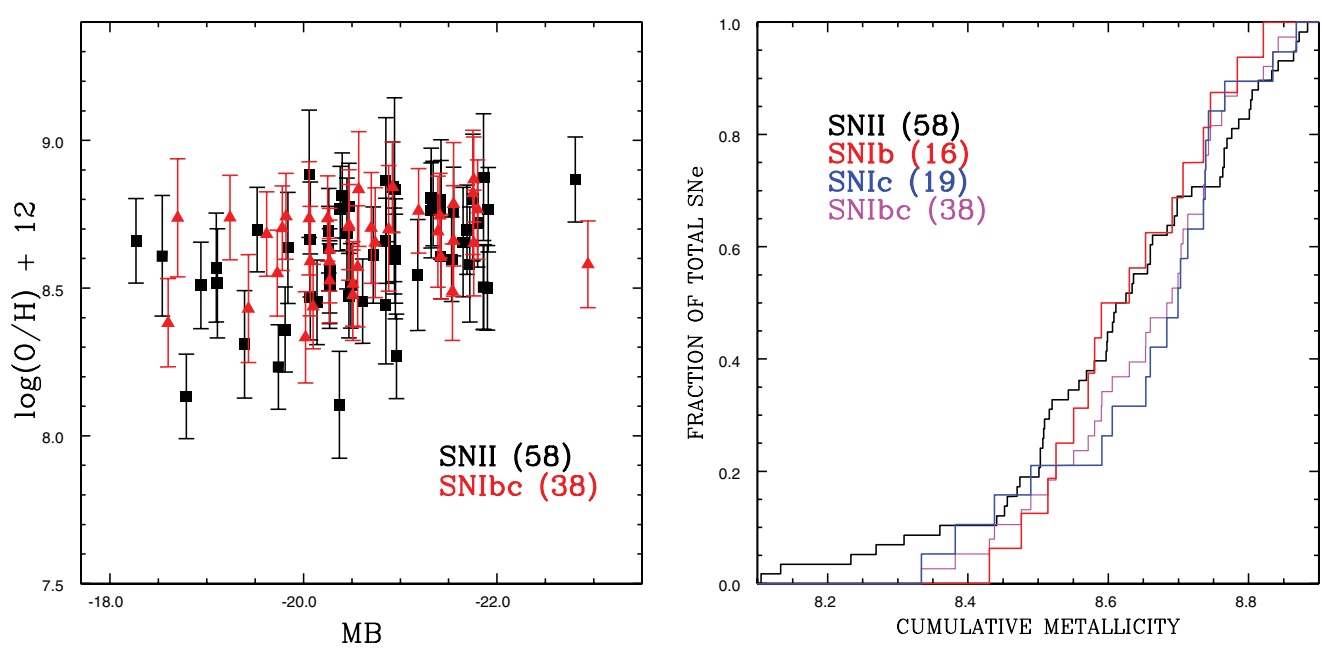

Figure 2. Left a): Host galaxy absolute $B$-band magnitudes against derived environment metallicities. SNII are shown in black squares while SNIbc are shown in red triangles. Right b): Cumulative metallicity distributions. The SNII distribution is plotted in black and the SNIbc distribution is shown in magenta. We also show the individual SNIb (red) and SNIc (blue) distributions.

mass progenitors (these results, plus discussion will be presented in Anderson et al. in preparation). On the sub-types plotted in Fig. 1b) we find the interesting result that both the 'impostors' and SNIIn show a lower degree of association with SF than the SNIIP. While there is likely to be a strong selection effect with respect to the 'impostors', for the SNIIn this implies that these events primarily arise from relatively low mass progenitors, contrary to general consensus. Finally, we find some suggestive constraints that both the SNIIL and SNIIb arise from higher mass progenitors than SIIP.

\section{Host HII region spectroscopy}

For $96 \mathrm{CC}$ SNe (58 SNII and 38 SNIbc) we have also obtained host HII region optical spectroscopy of their immediate environments which we use to determine region metallicities. To achieve this we use the diagnostics of Pettini \& Pagel (2004) which give gas-phase oxygen metallicities from emission line ratios. In Figure 2 we show the resulting distributions of each CC SN sample. Fig. 2a) plots the absolute $B$-band magnitude of host galaxies against derived environment metallicities. While the mean metallicity of SNIbc is higher than that of the SNII, this difference is not significant, and there is no clear offset between the distributions. In Fig. 2b) we display the cumulative metallicity distributions of the SNII, SNIb, SNIc and the combined SNIbc. While we see a metallicity trend going from the SNII in the lowest, through the SNIb to the SNIc within the highest metallicity regions, the difference between these distributions is statistically insignificant. Overall we see that SNIbc and SNII arise from similar metallicity environments, implying similar progenitor metallicities. While this is the only study thus far to derive 'direct' environment metallicities to probe differences between SNII and SNIbc events (a smaller sample was presented in Anderson et al. 2010), other recent results have been published on differences between SNIb, SNIc and LGRBs, finding different results (Leloudas et al. 2011, Modjaz et al. 2011, and talks at this symposium by the same authors). 


\section{Conclusions}

Through observations of the environments of CC SNe we have presented progenitor constraints. We find that the main SN types can be ordered into a sequence of increasing progenitor mass: SNIa-SNII-SNIb-SNIc. With respect to progenitor metallicity we find no significant difference between the SNII and SNIbc populations. This argues that mass is the dominant (over metallicity) progenitor characteristic that influences resulting SN type.

Acknowledgments J.A. acknowledges support from FONDECYT grant 3110142, and grant ICM P10-064-F (Millennium Center for Supernova Science), with input from 'Fondo de Innovacion para la Competitividad, del Ministerio de Economia, Fomento y Turismo de Chile'.

\section{References}

Anderson, J. P. \& James, P. A., 2008 MNRAS, 390, 1527

Anderson, J. P. et al., 2010 MNRAS, 407, 2660

Boissier, S. \& Prantzos, N., 2009, A\& $A, 503,137$

James, P. A. \& Anderson, J. P., 2006 A\&A, 453, 57

Kelly, P. L. \& Kirshner, R. P., 2011 arXiv, 1110.1377

Leloudas, G., et al., 2010 A 6 A, 518, 29

Leloudas, G., et al., $2011 A \mathscr{E} A, 530,95$

Modjaz, M., et al. 2011 ApJ, 731, 4

Pettini, M. \& Pagel, B. E. J., 2004 MNRAS, 348, 59

Prieto, J. L., Stanek, K. Z., \& Beacom, J. F. 2008, ApJ, 673, 999

Smartt, S. J., 2009, ARAA, 47, 63

\section{Discussion}

Crowther: Regarding the SNIIn results; if these are linked to Luminous Blue Variable (LBV) eruptions, locally LBVs span a wide range of mass with most shying away from HII regions (except notably Eta Carinae!)

ANDERSON: SNIIn show a lower association to HII regions than SNIIP. The (to-date) progenitors of SNIIP are 8-16 $\mathrm{M}_{\odot}$. Given that our results suggest that SNIIn arise from lower masses than SNIIP, LBV progenitors seem inconsistent with this picture.

Crowther: How would your statistics differ if you limited your sample volume, given that many SNIIP are faint and so inherently trace the lowest mass progenitors?

ANDERSON: We have split our sample by host recession velocity. If there were a significant effect with distance then we would expect NCR values to differ with distance. We do not observe this.

PERLEY: A significant fraction of Ics are probably dust-obscured. How do you think dust extinction -either impacting the images (dust-lanes) or the non-detection of some eventsmight affect your results?

AndERSON: SNIc are strongly associated with SF. If either $\mathrm{H} \alpha$ emission were being obscured, or SNIc were missed due to dust, this would only strengthen our result; more SNIc than we observe would be instrinsically associated with HII regions. 